\title{
Applications of an HIDS Theorem to the Existence of Fixed Point, Abstract Equilibria and Optimization Problems
}

\author{
Wei-Shih Du \\ Department of Mathematics, National Kaohsiung Normal University, Kaohsiung 824, Taiwan \\ Correspondence should be addressed to Wei-Shih Du, wsdu@nknucc.nknu.edu.tw
}

Received 16 June 2011; Accepted 18 July 2011

Academic Editor: Josip E. Pečarić

Copyright (c) 2011 Wei-Shih Du. This is an open access article distributed under the Creative Commons Attribution License, which permits unrestricted use, distribution, and reproduction in any medium, provided the original work is properly cited.

By applying hybrid inclusion and disclusion systems (HIDS), we establish several vectorial variants of system of Ekeland's variational principle on topological vector spaces, some existence theorems of system of parametric vectorial quasi-equilibrium problem, and an existence theorem of system of the Stampacchia-type vectorial equilibrium problem. As an application, a vectorial minimization theorem is also given. Moreover, we discuss some equivalence relations between our vectorial variant of Ekeland's variational principle, common fixed point theorem, and maximal element theorem.

\section{Introduction}

Let $X$ be a nonempty subset of a topological space (t.s., for short), and let $f: X \times X \rightarrow \mathbb{R}$ be a function with $f(x, x) \geq 0$ for all $x \in X$. Then the scalar equilibrium problem in the sense of Blum and Oettli [1] is to find $\bar{x} \in X$ such that $f(\bar{x}, y) \geq 0$ for all $y \in X$. The equilibrium problem was extensively investigated and generalized to the vectorial equilibrium problems for single-valued or multivalued maps and contains optimization problems, variational inequalities problems, saddle point problems, the Nash equilibrium problems, fixed point problems, complementary problems, bilevel problems, and semi-infinite problems as special cases and have some applications in mathematical program with equilibrium constraint; for detail one can refer to [1-4] and references therein.

The famous Ekeland's variational principle (EVP, for short) [5-7] is a forceful tool in various fields of applied mathematical analysis and nonlinear analysis. A number of generalizations in various different directions of these results for functions defined in metric (or quasimetric) spaces and more general in topological vector spaces have been investigated by several authors in the past; see [8-23] and references therein. It is wellknown that the original EVP is equivalent to Caristi's fixed point theorem, to Takahashi's nonconvex 
minimization theorem; and to the flower petal theorem for detail, see [14, 16-18, 20] and references therein. EVPs were extended to the vector case by using scalarization method and were applied to the study of efficiency (or approximative efficiency) and others; see, for example, $[3,8,9,12,13,15,23]$.

Recently, the author first studied the following mathematical model about hybrid inclusion and disclusion systems (HIDS, for short) [11]. Let $I$ be any index set. For each $i \in I$, let $Y_{i}$ be a nonempty closed convex subset of a Hausdorff topological vector space (t.v.s., for short) $V_{i}, H_{i} \subseteq Y_{i}, Y=\prod_{i \in I} Y_{i}, A_{i}: Y \multimap Y_{i}$, and let $T_{i}: Y \multimap Y_{i}$ be multivalued maps. Hybrid inclusion and disclusion systems (HIDS) are defined as follows:

$$
\text { (HIDS) Find } v=(v)_{i \in I} \in Y \quad \text { such that } v_{i} \in H_{i}
$$

$$
y_{i} \notin A_{i}(v), \quad \forall y_{i} \in T_{i}(v), \forall i \in I .
$$

In fact, HIDS contains several important problems as special cases. Let $X$ be a nonempty subset of a topological space $E$, and let $u \in X$ be given. For each $i \in I$, let $U_{i}$ and $Z_{i}$ be real t.v.s. with zero vector $\theta_{U_{i}}$ and $\theta_{Z_{i}}$, respectively.

Example 1.1. For each $i \in I$, let $F_{i}: X \times Y_{i} \rightarrow \mathbb{R}$ and $G_{i}: Y \times Y_{i} \rightarrow \mathbb{R}$ be functions. If $H_{i}$ and $A_{i}$ are defined as follows:

$$
\begin{gathered}
H_{i}=\left\{y_{i} \in Y_{i}: F_{i}\left(u, y_{i}\right) \leq 0\right\}, \\
A_{i}(y)=\left\{z_{i} \in Y_{i}: G_{i}\left(y, z_{i}\right) \leq 0\right\},
\end{gathered}
$$

then HIDS will reduce to the following system of hybrid scalar equilibrium problem $\left(P_{1}\right)$ :

$\left(P_{1}\right)$ Find $v=(v)_{i \in I} \in Y$ such that $F_{i}\left(u, v_{i}\right) \leq 0$ and $G_{i}\left(v, y_{i}\right)>0$ for all $y_{i} \in T_{i}(v)$ and for all $i \in I$.

Example 1.2. For each $i \in I$, let $F_{i}: X \times Y_{i} \multimap U_{i}$ and $G_{i}: Y \times Y_{i} \multimap Z_{i}$ be multivalued maps with nonempty values, and let $C_{i}$ and $D_{i}$ be nonempty subsets of $U_{i}$ and $Z_{i}$, respectively. If $H_{i}$ and $A_{i}$ are defined as follows:

$$
\begin{gathered}
H_{i}=\left\{y_{i} \in Y_{i}: F_{i}\left(u, y_{i}\right) \cap\left(-C_{i} \backslash\left\{\theta_{U_{i}}\right\}\right)=\emptyset\right\}, \\
A_{i}(y)=\left\{z_{i} \in Y_{i}: G_{i}\left(y, z_{i}\right) \cap\left(-D_{i} \backslash\left\{\theta_{Z_{i}}\right\}\right)=\emptyset\right\},
\end{gathered}
$$

then HIDS will reduce to the following problem $\left(P_{2}\right)$, which is an abstract equilibrium problem:

$\left(P_{2}\right)$ Find $v=(v)_{i \in I} \in Y$ such that $F_{i}\left(u, v_{i}\right) \cap\left(-C_{i} \backslash\left\{\theta_{U_{i}}\right\}\right)=\emptyset$ and $G_{i}\left(v, y_{i}\right) \cap\left(-D_{i} \backslash\right.$ $\left.\left\{\theta_{Z_{i}}\right\}\right) \neq \emptyset$ for all $y_{i} \in T_{i}(v)$ and for all $i \in I$.

Example 1.3. For each $i \in I$, let $F_{i}: Y_{i} \multimap U_{i}$ and $G_{i}: Y \times Y_{i} \multimap Z_{i}$ be multivalued maps. If $H_{i}$ and $A_{i}$ are defined as follows:

$$
\begin{gathered}
H_{i}=\left\{y_{i} \in Y_{i}: y_{i} \in F_{i}\left(y_{i}\right)\right\}, \\
A_{i}(y)=\left\{z_{i} \in Y_{i}: y \notin G_{i}\left(y, z_{i}\right)\right\},
\end{gathered}
$$


then HIDS will reduce to the following fixed point problem $\left(P_{3}\right)$ :

$\left(P_{3}\right)$ Find $v=(v)_{i \in I} \in Y$ such that $v_{i} \in F_{i}\left(v_{i}\right)$ and $v \in G_{i}\left(v, y_{i}\right)$ for all $y_{i} \in T_{i}(v)$ for all $i \in I$.

Example 1.4. For each $i \in I$, let $F_{i}: X \times Y_{i} \multimap U_{i}$ and $G_{i}: Y \times Y_{i} \multimap Z_{i}$ be multivalued maps with nonempty values. If $H_{i}$ and $A_{i}$ are defined as follows:

$$
\begin{gathered}
H_{i}=\left\{y_{i} \in Y_{i}: \theta_{U_{i}} \notin F_{i}\left(u, y_{i}\right)\right\}, \\
A_{i}(y)=\left\{z_{i} \in Y_{i}: \theta_{Z_{i}} \notin G_{i}\left(y, z_{i}\right)\right\},
\end{gathered}
$$

then HIDS will reduce to the following system of mixed type of parametric variational inclusion and disclusion problem $\left(P_{4}\right)$ :

$\left(P_{4}\right)$ Find $v=(v)_{i \in I} \in Y$ such that $\theta_{U_{i}} \notin F_{i}\left(u, v_{i}\right)$ and $\theta_{Z_{i}} \in G_{i}\left(v, y_{i}\right)$ for all $y_{i} \in T_{i}(v)$ and for all $i \in I$.

In this paper, we study the existence theorems of system of parametric vectorial quasi-equilibrium problems, vectorial variants of system of Ekeland's variational principle (VSEVP, for short), and the existence theorems of system of the Stampacchia-type vectorial equilibrium problem by using an HIDS theorem established by the author [11]. Our results improve and generalize some theorems in [19] to the vector case. Till now, to my knowledge, there are extremely few results about Stampacchia-type vectorial equilibrium problem in the literature. The existence of VSEVP and the Stampacchia-type vectorial equilibrium problem are established by applying the HIDS theorem without the use of any scalarization method. So our results are completely different from [3, 8, 9, 12, 13, 15, 23]. As an application, a vectorial minimization theorem is also proved. Moreover, we prove some equivalence relations between our VSEVP, common fixed point theorem, and maximal element theorem.

\section{Preliminaries}

Let $A$ and $B$ be nonempty sets. A multivalued map $T: A \multimap B$ is a function from $A$ to the power set $2^{B}$ of $B$. We denote $T(A)=\bigcup\{T(x): x \in A\}$ and let $T^{-}: B \multimap A$ be defined by the condition that $x \in T^{-}(y)$ if and only if $y \in T(x)$. We recall that a point $x \in A$ is a maximal element of $T: A \rightarrow 2^{B}$ if $T(x)=\emptyset$. Let $X$ be a linear space with zero vector $\theta$. A nonempty subset $C$ of $X$ is called a convex cone if $C+C \subseteq C$ and $\lambda C \subseteq C$ for all $\lambda \geq 0$. A cone $C$ in $X$ is pointed if $C \cap(-C)=\{\theta\}$. Let $Z$ be a real t.v.s., and let $D$ be a proper convex cone in $Z$, and $A \subseteq Z$. A point $\bar{y} \in A$ is called a vectorial minimal point of $A$ with respect to $D$ if for any $y \in A, y-\bar{y} \notin-D \backslash\{\theta\}$. The set of vectorial minimal point of $A$ is denoted by $\operatorname{Min}_{D} A$. The convex hull of $A$ and the closure of $A$ are denoted by co $A$ and cl $A$, respectively.

Definition 2.1. Let $X$ and $Y$ be linear spaces, and let $C$ be a proper convex cone in $Y$. A map $f: X \multimap Y$ is called $C$-convex if for any $x_{1}, x_{2} \in X$ and $\lambda \in[0,1]$, one has

$$
\lambda f\left(x_{1}\right)+(1-\lambda) f\left(x_{2}\right)-f\left(\lambda x_{1}+(1-\lambda) x_{2}\right) \subseteq C .
$$

Clearly, if $f_{1}$ and $f_{2}$ are $C$-convex and $\alpha \geq 0$, then $\alpha f_{1}$ and $f_{1}+f_{2}$ are $C$-convex. 
Definition 2.2. Let $X$ be a nonempty convex subset of a vector space $E$, let $Y$ be a nonempty convex subset of a vector space $V$, and let $Z$ be a real t.v.s. Let $F: X \times Y \multimap Z$ and $C: X \multimap Z$ be multivalued maps such that for each $x \in X, C(x)$ is a nonempty closed convex cone. For each fixed $x \in X, y \multimap F(x, y)$ is called $C(x)$-quasiconvex if for any $y_{1}, y_{2} \in Y$ and $\lambda \in[0,1]$, one has either

$$
F\left(x, y_{1}\right) \subseteq F\left(x, \lambda y_{1}+(1-\lambda) y_{2}\right)+C(x)
$$

or

$$
F\left(x, y_{2}\right) \subseteq F\left(x, \lambda y_{1}+(1-\lambda) y_{2}\right)+C(x)
$$

Now, we define the concept of vectorial upper and lower semicontinuous on t.v.s.

Definition 2.3. Let $X$ be a t.s., let $Y$ be a t.v.s. with zero vector $\theta$, and let $C \neq\{\theta\}$ be a pointed convex cone in $Y$. A map $f: X \rightarrow Y$ is said to be

(i) vectorial lower semicontinuous with respect to $C$ (C-v.l.s.c., for short) at $x_{0} \in X$ if for any $a \in C \backslash\{\theta\}$, there exists an open neighborhood $N\left(x_{0}\right)$ of $x_{0}$ such that $f(y)-$ $f\left(x_{0}\right)+a \in C \backslash\{\theta\}$ for all $y \in N\left(x_{0}\right)$,

(ii) vectorial upper semicontinuous with respect to $C$ (C-v.u.s.c., for short) at $x_{0} \in X$ if for any $a \in C \backslash\{\theta\}$, there exists an open neighborhood $N\left(x_{0}\right)$ of $x_{0}$ such that $f\left(x_{0}\right)-$ $f(y)+a \in C \backslash\{\theta\}$ for all $y \in N\left(x_{0}\right)$.

The function $f$ is called $C$-v.l.s.c. (resp., $C$-v.u.s.c.) on $X$ if $f$ is $C$-v.l.s.c. (resp., $C$-v.u.s.c.) at every point of $X$.

Proposition 2.4. Let $X$ be a t.s., let $Y$ be a t.v.s with zero vector $\theta$, and let $C \neq\{\theta\}$ be a pointed convex cone in $Y$. Let $f, g: X \rightarrow Y$ be maps and $\gamma \geq 0$. Then the following hold:

(a) $f$ is C-v.u.s.c. (resp., C-v.l.s.c.) on $X \Longleftrightarrow-f$ is C-v.l.s.c. (resp., C-v.u.s.c.) on $X$;

(b) if $f$ and $g$ are C-v.u.s.c. (resp., C-v.l.s.c.) on $X$, then $\gamma f$ and $f+g$ are C-v.u.s.c. (resp., C-v.l.s.c.) on $X$;

(c) if $f$ is $C$-v.u.s.c. on $X$, then

(i) $\{x \in X: \lambda-f(x) \in C \backslash\{\theta\}\}$ is open in $X$ for all $\lambda \in Y$,

(ii) $\{x \in X: \lambda-f(x) \notin C \backslash\{\theta\}\}$ is closed in $X$ for all $\lambda \in Y$;

(d) if $f$ is C-v.l.s.c. on $X$, then

(iii) $\{x \in X: f(x)-\lambda \in C \backslash\{\theta\}\}$ is open in $X$ for all $\lambda \in Y$,

(iv) $\{x \in X: f(x)-\lambda \notin C \backslash\{\theta\}\}$ is closed in $X$ for all $\lambda \in Y$.

Proof. Clearly, (a) and (b) hold from definition. To prove (c), it suffices to show (i). Suppose that $f$ is $C$-v.u.s.c. on $X$. Let $\lambda \in Y$ and $x_{0} \in\{x \in X: \lambda-f(x) \in C \backslash\{\theta\}\}$. Then $\alpha:=\lambda-f\left(x_{0}\right) \in$ $C \backslash\{\theta\}$. Since $f$ is $C$-v.u.s.c. at $x_{0}$, there exists an open neighborhood $N\left(x_{0}\right)$ of $x_{0}$ such that

$$
\curlywedge-f(y)=f\left(x_{0}\right)-f(y)+\alpha \in C \backslash\{\theta\}
$$


for all $y \in N\left(x_{0}\right)$. Hence $\{x \in X: \lambda-f(x) \in C \backslash\{\theta\}\}$ is an open set in $X$ and (i) is proved. Obviously, (ii) is immediate from (i). It is easy to see that conclusion (d) follows from (a) and (c).

Remark 2.5. Let $X$ be a t.v.s. and $\alpha \in X$ with $\alpha \neq 0_{X}$, where $0_{X}$ is the origin of $X$. Let $f: X \rightarrow$ $L_{\alpha}=\{\gamma \alpha: \gamma \in \mathbb{R}\}$ be a map. Hence in conclusion (c) (resp., (d)) of Proposition 2.4, we have

$$
\begin{aligned}
& f \text { is } C \text {-v.u.s.c. on } X \Longleftrightarrow(\text { i) } \Longleftrightarrow \text { (ii) } \\
& \text { (resp., } f \text { is } C \text {-v.l.s.c. on } X \Longleftrightarrow \text { (iii) } \Longleftrightarrow \text { (iv)). }
\end{aligned}
$$

In particular, if $X=\mathbb{R}$ (the set of real numbers) and $C=[0, \infty)$, then the $C$-v.l.s.c. (resp. $C$-v.u.s.c.) function $f: X \rightarrow \mathbb{R}$ is l.s.c. (resp. u.s.c.) in usual.

The concept of $C$-vectorial $\ell$-function and $C$-vectorial quasi-distance on topological spaces are introduced as follows.

Definition 2.6. Let $X$ and $E$ be t.v.s., let $\theta$ be the zero vector of $E$, and let $C \neq\{\theta\}$, a pointed convex cone in $E$. A map $p: X \times X \rightarrow E$ is called

(a) a $C$-vectorial $\ell$-function ( $\ell_{C}$-function, for short) if the following are satisfied:

(VL1) $p(x, x) \in C$ for all $x \in X$;

$(V L 2)$ for any $x \in X, p(x, \cdot)$ is $C$-convex;

(VL3) for any $y \in X, p(\cdot, y)$ is $C$-v.u.s.c.

(b) a C-vectorial quasi-distance if the following are satisfied:

(VQD1) $p(x, x) \in C$ for all $x \in X$;

$(V Q D 2) p(x, y)+p(y, z) \in p(x, z)+C$ for any $x, y, z \in X$

(VQD3) for any $x \in X, p(x, \cdot)$ is $C$-convex and $C$-v.l.s.c.;

$(V Q D 4)$ for any $y \in X, p(\cdot, y)$ is $C$-v.u.s.c.

If $E=(-\infty, \infty]$ and letting $C=[0, \infty)$ be in (a) and (b), then the function $p$ : $X \times X \rightarrow(-\infty, \infty]$ is called a $\ell$-function and quasi-distance, respectively, introduced by Lin and Du [19]. For examples and results of $\ell$-function and quasi-distance, one can see [19].

Remark 2.7. (a) Obviously, a $C$-vectorial quasi-distance is a $\ell_{C}$-function, but the reverse is not true;

(b) if $p_{1}$ and $p_{2}$ are $C$-vectorial quasi-distances (resp., $\ell_{C}$-functions) and $\alpha \geq 0$, then $\alpha p_{1}$ and $p_{1}+p_{2}$ are $C$-vectorial quasi-distances (resp., $\ell_{C}$-functions);

(c) if $f: X \rightarrow E$ is a $C$-v.l.s.c. and $C$-convex function, then the function $p: X \times X \rightarrow E$ defined by $p(x, y)=f(y)-f(x)$ is a $C$-vectorial quasi-distance.

Lemma 2.8 (see $[24,25])$. Let $X$ and $Y$ be the Hausdorff topological spaces, and let $T: X \multimap Y$ be $a$ multivalued map. Then $T$ is l.s.c. at $x \in X$ if and only if for any $y \in T(x)$ and for any net $\left\{x_{\alpha}\right\}$ in $X$ converging to $x$, there exists a subnet $\left\{x_{\phi(\lambda)}\right\}_{\lambda \in \Lambda}$ of $\left\{x_{\alpha}\right\}$ and a net $\left\{y_{\lambda}\right\}_{\lambda \in \Lambda}$ with $y_{\lambda} \rightarrow y$ such that $y_{\lambda} \in T\left(x_{\phi(\lambda)}\right)$ for all $\lambda \in \Lambda$. 


\section{The Existence of System of VSEVP and Abstract Equilibrium Problems}

The following existence theorem for the solution of HIDS was established in [11].

Theorem 3.1 (HIDS theorem [11]). Let I be any index set. For each $i \in I$, let $Y_{i}$ be a nonempty closed convex subset of a Hausdorff t.v.s. $V_{i}$. Let $X$ be a nonempty subset of a topological space $E$, $Y=\prod_{i \in I} Y_{i}$ and $u \in X$. For each $i \in I$, let $H_{i}$ be a nonempty closed subset of $Y_{i}$, let $A_{i}: Y \multimap Y_{i}$ be a multivalued map, and let $T_{i}: Y \multimap Y_{i}$ be a multivalued map with nonempty values. For each $i \in I$, suppose that the following conditions are satisfied:

(i) for each $y=\left(y_{i}\right)_{i \in I} \in Y, y_{i} \notin A_{i}(y)$;

(ii) for each $y \in Y, \operatorname{co} T_{i}(y) \subseteq H_{i}$ and $A_{i}(y)$ is convex;

(iii) for each $z_{i} \in Y_{i}, T_{i}^{-}\left(z_{i}\right)$ and $A_{i}^{-}\left(z_{i}\right)$ are open in $Y$;

(iv) there exist a nonempty compact subset $K$ of $Y$ and a nonempty compact convex subset $M_{i}$ of $Y_{i}$ for each $i \in I$ such that for each $y \in Y \backslash K$ there exist $j \in I$ and $z_{j} \in M_{j} \cap T_{j}(y) \cap A_{j}(y)$.

Then there exists $v=\left(v_{i}\right)_{i \in I} \in Y$ such that for each $i \in I, v_{i} \in H_{i}$ and $y_{i} \notin A_{i}(v)$ for all $y_{i} \in T_{i}(v)$.

Example 3.2. Let $X$ and $Y$ be Hausdorff t.v.s., let $U$ be a real t.v.s. with its zero vector $\theta$, and $u \in X$.

(a) Let $F: X \times Y \multimap U$ be a multivalued map with nonempty values such that there exists $w=w(u) \in Y$ such that $\theta \in F(u, w)$ and the map $y \multimap F(u, y)$ is closed. Then it is easy to see that $H=\{y \in Y: \theta \in F(u, y)\}$ is a nonempty closed subset of $Y$.

(b) Let $G: Y \times Y \multimap U$ be a multivalued map with nonempty values and $W$ be a nonempty open set in $U$. Suppose that

(i) for each $y \in Y, \theta \notin G(y, y)+W$,

(ii) for each $y \in Y, G(y, \cdot)$ is $\{\theta\}$-quasiconvex and for each $z \in Y, G(\cdot, z)$ is l.s.c.

Let $A: Y \multimap Y$ be defined by

$$
A(y)=\{z \in Y: \theta \in G(y, z)+W\} .
$$

Then $y \notin A(y)$ for each $y \in Y$. We claim that $A^{-}(z)$ is open in $Y$ for each $z \in Y$. Indeed, let $z \in Y$ be given, and let $y \in \operatorname{cl}\left(Y \backslash A^{-}(z)\right)$. Then there exists a net $\left\{y_{\alpha}\right\}_{\alpha \in \Lambda}$ in $Y \backslash A^{-}(z)$ such that $y_{\alpha} \rightarrow y$. Thus we have $\theta \notin G\left(y_{\alpha}, z\right)+W$ or $G\left(y_{\alpha}, z\right) \subseteq U \backslash W$. Clearly, $y \in Y$. For any $w \in G(y, z)$, since $G(\cdot, z)$ is 1.s.c. at $y$ and $y_{\alpha} \rightarrow y$, by Lemma 2.8, there exists a net $\left\{w_{\alpha}\right\}$ with $w_{\alpha} \rightarrow w$ such that $w_{\alpha} \in G\left(y_{\alpha}, z\right) \subseteq U \backslash W$. Since $U \backslash W$ is closed, $w \in U \backslash W$. So $G(y, z) \subseteq U \backslash W$. It implies $\operatorname{cl}\left(Y \backslash A^{-}(z)\right)=Y \backslash A^{-}(z)$, and hence $A^{-}(z)$ is open in $Y$. Next, we show that for each $y \in Y, A(y)$ is convex. Let $a, b \in A(y)$. Then $\theta \in G(y, a)+W$ and $\theta \in G(y, b)+W$. For any $\lambda \in[0,1]$, let $e_{\lambda}:=\lambda a+(1-\lambda) b \in Y$. Suppose to the contrary that there exists $\lambda_{0} \in(0,1)$ such that $\theta \notin G\left(y, e_{\lambda_{0}}\right)+W$. By the $\{\theta\}$-quasiconvexity of $G(y, \cdot)$, either

$$
\theta \in G(y, a)+W \subseteq G\left(y, e_{\lambda_{0}}\right)+W
$$

or

$$
\theta \in G(y, b)+W \subseteq G\left(y, e_{\lambda_{0}}\right)+W
$$

which leads to a contradiction. Hence for each $y \in Y, A(y)$ is convex. 
Applying Theorem 3.1, we establish the following existence theorem of system of parametric vectorial quasi-equilibrium problem.

Theorem 3.3. Let $I$ be any index set. For each $i \in I$, let $X_{i}$ be a nonempty Hausdorff t.v.s., and let $C_{i} \neq\{\theta\}$ be a pointed convex cone in a t.v.s. E with zero vector $\theta$. Let $X=\prod_{i \in I} X_{i}$. For each $i \in I$, let $p_{i}, q_{i}: X_{i} \times X_{i} \rightarrow E$ be $C_{i}$-vectorial quasi-distances and let $T_{i}: X \multimap X_{i}$ be a multivalued map with nonempty values. Let $u=\left(u_{i}\right)_{i \in I} \in X$ with $p_{i}\left(u_{i}, u_{i}\right)=q_{i}\left(u_{i}, u_{i}\right)=\theta$ for all $i \in I$. For each $i \in I$, suppose that the following conditions are satisfied:

(i) for each $y \in X, \operatorname{co} T_{i}(y) \subseteq\left\{x_{i} \in X_{i}: q_{i}\left(u_{i}, x_{i}\right) \notin C_{i} \backslash\{\theta\}\right\}$ and $T_{i}^{-}\left(z_{i}\right)$ is open for all $z_{i} \in X_{i}$

(ii) there exist a nonempty compact subset $K$ of $X$ and a nonempty compact convex subset $M_{i}$ of $X_{i}$ for each $i \in I$ such that for each $y \in X \backslash K$ there exist $j \in I$ and $z_{j} \in M_{j} \cap T_{j}(y)$ such that $p_{j}\left(y_{j}, z_{j}\right) \in-C_{j} \backslash\{\theta\}$.

Then there exists $v=\left(v_{i}\right)_{i \in I} \in X$ such that for each $i \in I$, one has

(a) $q_{i}\left(u_{i}, v_{i}\right) \notin C_{i} \backslash\{\theta\}$,

(b) $p_{i}\left(v_{i}, x_{i}\right) \notin-C_{i} \backslash\{\theta\}$ for all $x_{i} \in T_{i}(v)$.

Proof. For each $i \in I$, define $F_{i}: X \times X_{i} \multimap E$ and $G_{i}: X \times X \times X_{i} \multimap E$ by

$$
\begin{gathered}
F_{i}\left(x, y_{i}\right)=-q_{i}\left(x_{i}, y_{i}\right)+C_{i} \backslash\{\theta\}, \quad \forall\left(x, y_{i}\right) \in X \times X_{i}, \\
G\left(t, x, y_{i}\right)=p_{i}\left(x_{i}, y_{i}\right)+C_{i} \backslash\{\theta\}, \quad \forall\left(t, x, y_{i}\right) \in X \times X \times X_{i},
\end{gathered}
$$

respectively. Thus, for each $i \in I$, we let

$$
H_{i}:=\left\{x_{i} \in X_{i}: \theta \notin F_{i}\left(u, x_{i}\right)\right\}=\left\{x_{i} \in X_{i}: q_{i}\left(u_{i}, x_{i}\right) \notin C_{i} \backslash\{\theta\}\right\},
$$

and let $A_{i}: X \multimap X_{i}$ be defined by

$$
\begin{aligned}
A_{i}(x) & =\left\{y_{i} \in X_{i}: \theta \in G_{i}\left(u, x, y_{i}\right)\right\} \\
& =\left\{y_{i} \in X_{i}: p_{i}\left(x_{i}, y_{i}\right) \in-C_{i} \backslash\{\theta\}\right\}, \quad \forall x=\left(x_{i}\right)_{i \in I} \in X .
\end{aligned}
$$

Clearly, for each $i \in I, \theta \notin G_{i}\left(u, x, x_{i}\right)$ for all $x=\left(x_{i}\right)_{i \in I} \in X$, and hence $x_{i} \notin A_{i}(x)$ for all $x=\left(x_{i}\right)_{i \in I} \in X$. By the $C_{i}$-vectorial lower semicontinuity of $q_{i}\left(u_{i}, \cdot\right), H_{i}$ is a nonempty closed subset of $X_{i}$. By (i), for each $x \in X, \operatorname{co} T_{i}(x) \subseteq H_{i}$. For each $y_{i} \in X_{i}$, by the vectorial upper semicontinuity of $p_{i}\left(\cdot, y_{i}\right)$, the set $\left\{x_{i} \in X_{i}: p_{i}\left(x_{i}, y_{i}\right) \in-C_{i} \backslash\{\theta\}\right\}$ is open in $X_{i}$, and hence $A_{i}^{-}\left(y_{i}\right)=\left\{x_{i} \in X_{i}: q_{i}\left(x_{i}, y_{i}\right) \in-C_{i} \backslash\{\theta\}\right\} \times \prod_{j \neq i} X_{j}$ is open in $X$. For each $x \in X, A_{i}(x)$ is convex from the $C_{i}$-convexity of $p_{i}\left(x_{i}, \cdot\right)$ for all $i \in I$. By (ii), there exist a nonempty compact subset $K$ of $X$ and a nonempty compact convex subset $M_{i}$ of $X_{i}$ for each $i \in I$ such that for each $y \in X \backslash K$ there exist $j \in I$ and $z_{j} \in M_{j} \cap T_{j}(y) \cap A_{j}(y)$. Therefore all the conditions of Theorem 3.1 are satisfied. Applying Theorem 3.1, there exists $v=\left(v_{i}\right)_{i \in I} \in X$ such that:

(1) $q_{i}\left(u_{i}, v_{i}\right) \notin C_{i} \backslash\{\theta\}$,

(2) $p_{i}\left(v_{i}, x_{i}\right) \notin-C_{i} \backslash\{\theta\}$ for all $x_{i} \in T_{i}(v)$. 
Theorem 3.4. Let $I, X, X, C_{i}, E, \theta, T_{i}$, and $p_{i}$ be the same as in Theorem 3.3. Let $u=\left(u_{i}\right)_{i \in I} \in X$ with $p_{i}\left(u_{i}, u_{i}\right)=\theta$ for all $i \in I$. For each $i \in I$, suppose that the following conditions are satisfied:

(i) for each $y \in X, \operatorname{co} T_{i}(y) \subseteq\left\{x_{i} \in X_{i}: p_{i}\left(u_{i}, x_{i}\right) \notin C_{i} \backslash\{\theta\}\right\}$ and $T_{i}^{-}\left(z_{i}\right)$ is open for all $z_{i} \in X_{i}$;

(ii) there exist a nonempty compact subset $K$ of $X$ and a nonempty compact convex subset $M_{i}$ of $X_{i}$ for each $i \in I$ such that for each $y \in X \backslash K$ there exist $j \in I$ and $z_{j} \in M_{j} \cap T_{j}(y)$ such that $p_{j}\left(y_{j}, z_{j}\right) \in-C_{j} \backslash\{\theta\}$.

Then there exists $v=\left(v_{i}\right)_{i \in I} \in X$ such that for each $i \in I$, one has

(a) $p_{i}\left(u_{i}, v_{i}\right) \notin C_{i} \backslash\{\theta\}$,

(b) $p_{i}\left(v_{i}, x_{i}\right) \notin-C_{i} \backslash\{\theta\}$ for all $x_{i} \in T_{i}(v) \cup\left\{z_{i} \in X_{i}: p_{i}\left(u_{i}, z_{i}\right) \in C_{i} \backslash\{\theta\}\right\}$.

Proof. For each $i \in I$, let $q_{i}: X_{i} \times X_{i} \rightarrow E$ be defined by $q_{i}=p_{i}$. By Theorem 3.3, there exists $v=\left(v_{i}\right)_{i \in I} \in X$ such that for each $i \in I$, we have

(1) $p_{i}\left(u_{i}, v_{i}\right) \notin C_{i} \backslash\{\theta\}$,

(2) $p_{i}\left(v_{i}, x_{i}\right) \notin-C_{i} \backslash\{\theta\}$ for all $x_{i} \in T_{i}(v)$.

We claim that for each $i \in I, p_{i}\left(v_{i}, x_{i}\right) \notin-C_{i} \backslash\{\theta\}$ for all $x_{i} \in\left\{z_{i} \in X_{i}: p_{i}\left(u_{i}, z_{i}\right) \in\right.$ $\left.C_{i} \backslash\{\theta\}\right\}$. For each $x_{i} \in\left\{z_{i} \in X_{i}: p_{i}\left(u_{i}, z_{i}\right) \in C_{i} \backslash\{\theta\}\right\}$, we have $p_{i}\left(u_{i}, x_{i}\right) \in C_{i} \backslash\{\theta\}$. If $p_{i}\left(v_{i}, x_{i}\right) \in-C_{i} \backslash\{\theta\}$, then, by (VQD2) (in Definition 2.6(b)), we obtain

$$
p_{i}\left(u_{i}, v_{i}\right) \in p_{i}\left(u_{i}, x_{i}\right)-p_{i}\left(v_{i}, x_{i}\right)+C_{i} \subseteq C_{i} \backslash\{\theta\},
$$

which contradict with (1). Hence $p_{i}\left(v_{i}, x_{i}\right) \notin-C_{i} \backslash\{\theta\}$ for all $x_{i} \in T_{i}(v) \cup\left\{z_{i} \in X_{i}: p_{i}\left(u_{i}, z_{i}\right) \in\right.$ $\left.C_{i} \backslash\{\theta\}\right\}$, and the proof is completed.

Remark 3.5. (i) Let $X$ be a nonempty Hausdorff t.v.s., and let $C \neq\{\theta\}$ be a nonempty pointed convex cone in a t.v.s. $E$ with zero vector $\theta$. If a map $p: X \times X \rightarrow E$ satisfies that for each $x \in X$, $p(x, \cdot)$ is $C$-quasiconvex, then for each $u \in X$ with $p(u, u)=\theta, W_{u}:=\{x \in X: p(u, x) \notin C \backslash\{\theta\}\}$ is a nonempty convex subset in $X$.

(ii) In Theorem 3.3, if for each $i \in I, W_{i}:=\left\{x_{i} \in X_{i}: p_{i}\left(u_{i}, x_{i}\right) \notin C_{i} \backslash\{\theta\}\right\}$ is convex and $T_{i}(x)=W_{i}$ for all $x \in X$, then conclusion (b) can be replaced with conclusion (b)', where

(b) $p_{i}\left(v_{i}, x_{i}\right) \notin-C_{i} \backslash\{\theta\}$, for all $x_{i} \in X_{i}$.

Since the sum of two $C_{i}$-vectorial quasi-distances is also a $C_{i}$-vectorial quasi-distance, the following results related with system of VSEVP for vectorial quasi-distances in a Hausdorff t.v.s. are immediate from Theorem 3.3.

Theorem 3.6. Let $I, X, X, C_{i}, E, \theta, T_{i}$, and $p_{i}$ be the same as in Theorem 3.3. Let $u=\left(u_{i}\right)_{i \in I} \in X$ with $p_{i}\left(u_{i}, u_{i}\right)=\theta$ for all $i \in I$. For each $i \in I$, let $f_{i}: X_{i} \rightarrow E$ be a $C_{i}$-v.l.s.c. and $C_{i}$-convex function and suppose that the following conditions are satisfied:

(i) for each $y \in X, \operatorname{co} T_{i}(y) \subseteq\left\{x_{i} \in X_{i}: f_{i}\left(x_{i}\right)-f_{i}\left(u_{i}\right) \notin C_{i} \backslash\{\theta\}\right\}$ and $T_{i}^{-}\left(z_{i}\right)$ is open for all $z_{i} \in X_{i}$

(ii) there exist a nonempty compact subset $K$ of $X$ and a nonempty compact convex subset $M_{i}$ of $X_{i}$ for each $i \in I$ such that for each $y \in X \backslash K$ there exist $j \in I$ and $z_{j} \in M_{j} \cap T_{j}(y)$ such that $p_{j}\left(y_{j}, z_{j}\right)+f_{j}\left(z_{j}\right)-f_{j}\left(y_{j}\right) \in-C_{j} \backslash\{\theta\}$. 
Then there exists $v=\left(v_{i}\right)_{i \in I} \in X$ such that for each $i \in I$, one has

(a) $f_{i}\left(v_{i}\right)-f_{i}\left(u_{i}\right) \notin C_{i} \backslash\{\theta\}$,

(b) $p_{i}\left(v_{i}, x_{i}\right)+f_{i}\left(x_{i}\right)-f_{i}\left(v_{i}\right) \notin-C_{i} \backslash\{\theta\}$ for all $x_{i} \in T_{i}(v)$. result.

In Theorem 3.6, if $E=(-\infty, \infty]$ and $C_{i}=[0, \infty)$ for all $i \in I$, then we have the following

Corollary 3.7. Let $I, X_{i}, X$, and $T_{i}$ be the same as in Theorem 3.3. For each $i \in I$, let $f_{i}: X_{i} \rightarrow$ $(-\infty, \infty]$ be a l.s.c. and convex function and let $p_{i}: X_{i} \times X_{i} \rightarrow(-\infty, \infty]$ be a quasi-distance. Let $u=\left(u_{i}\right)_{i \in I} \in X$ with $p_{i}\left(u_{i}, u_{i}\right)=0$ for all $i \in I$. Suppose that the following conditions are satisfied:

(i) for each $y \in X, \operatorname{co} T_{i}(y) \subseteq\left\{x_{i} \in X_{i}: f_{i}\left(x_{i}\right) \leq f_{i}\left(u_{i}\right)\right\}$ and $T_{i}^{-}\left(z_{i}\right)$ is open for all $z_{i} \in X_{i}$;

(ii) there exist a nonempty compact subset $K$ of $X$ and a nonempty compact convex subset $M_{i}$ of $X_{i}$ for each $i \in I$ such that for each $y \in X \backslash K$ there exist $j \in I$ and $z_{j} \in M_{j} \cap T_{j}(y)$ such that $p_{j}\left(y_{j}, z_{j}\right)<f_{j}\left(y_{j}\right)-f_{j}\left(z_{j}\right)$.

Then there exists $v=\left(v_{i}\right)_{i \in I} \in X$ such that for each $i \in I$, one has

(a) $f_{i}\left(v_{i}\right) \leq f_{i}\left(u_{i}\right)$,

(b) $p_{i}\left(v_{i}, x_{i}\right) \geq f_{i}\left(v_{i}\right)-f_{i}\left(x_{i}\right)$ for all $x_{i} \in T_{i}(v)$.

Theorem 3.8. Let $I, X, X, C_{i}, E, \theta, T_{i}$, and $p_{i}$ be the same as in Theorem 3.3. Let $u=\left(u_{i}\right)_{i \in I} \in X$ with $p_{i}\left(u_{i}, u_{i}\right)=\theta$ for all $i \in I$. For each $i \in I$, let $f_{i}: X_{i} \rightarrow E$ be a $C_{i}$-v.l.s.c. and $C_{i}$-convex function and suppose that the following conditions are satisfied:

(i) for each $y \in X, \operatorname{co} T_{i}(y) \subseteq\left\{x_{i} \in X_{i}: p_{i}\left(u_{i}, x_{i}\right)+f_{i}\left(x_{i}\right)-f_{i}\left(u_{i}\right) \notin C_{i} \backslash\{\theta\}\right\}$ and $T_{i}^{-}\left(z_{i}\right)$ is open for all $z_{i} \in X_{i}$;

(ii) there exist a nonempty compact subset $K$ of $X$ and a nonempty compact convex subset $M_{i}$ of $X_{i}$ for each $i \in I$ such that for each $y \in X \backslash K$ there exist $j \in I$ and $z_{j} \in M_{j} \cap T_{j}(y)$ such that $p_{j}\left(y_{j}, z_{j}\right)+f_{j}\left(z_{j}\right)-f_{j}\left(y_{j}\right) \in-C_{j} \backslash\{\theta\}$.

Then there exists $v=\left(v_{i}\right)_{i \in I} \in X$ such that for each $i \in I$, one has

(a) $p_{i}\left(u_{i}, v_{i}\right)+f_{i}\left(v_{i}\right)-f_{i}\left(u_{i}\right) \notin C_{i} \backslash\{\theta\}$,

(b) $p_{i}\left(v_{i}, x_{i}\right)+f_{i}\left(x_{i}\right)-f_{i}\left(v_{i}\right) \notin-C_{i} \backslash\{\theta\}$ for all $x_{i} \in T_{i}(v) \cup\left\{z_{i} \in X_{i}: p_{i}\left(u_{i}, z_{i}\right)+f_{i}\left(z_{i}\right)-\right.$ $\left.f_{i}\left(u_{i}\right) \in C_{i} \backslash\{\theta\}\right\}$.

Corollary 3.9. Let $I, X_{i}, X$, and $T_{i}$ be the same as in Theorem 3.3. For each $i \in I$, let $f_{i}: X_{i} \rightarrow$ $(-\infty, \infty]$ be a l.s.c. and convex function and $p_{i}: X_{i} \times X_{i} \rightarrow(-\infty, \infty]$ be a quasi-distance. Let $u=\left(u_{i}\right)_{i \in I} \in X$ with $p_{i}\left(u_{i}, u_{i}\right)=0$ for all $i \in I$. Suppose that the following conditions are satisfied:

(i) for each $y \in X, \operatorname{co} T_{i}(y) \subseteq\left\{x_{i} \in X_{i}: p_{i}\left(u_{i}, x_{i}\right) \leq f_{i}\left(u_{i}\right)-f_{i}\left(x_{i}\right)\right\}$ and $T_{i}^{-}\left(z_{i}\right)$ is open for all $z_{i} \in X_{i}$

(ii) there exist a nonempty compact subset $K$ of $X$ and a nonempty compact convex subset $M_{i}$ of $X_{i}$ for each $i \in I$ such that for each $y \in X \backslash K$ there exist $j \in I$ and $z_{j} \in M_{j} \cap T_{j}(y)$ such that $p_{j}\left(y_{j}, z_{j}\right)<f_{j}\left(y_{j}\right)-f_{j}\left(z_{j}\right)$. 
Then there exists $v=\left(v_{i}\right)_{i \in I} \in X$ such that for each $i \in I$, one has

(a) $p_{i}\left(u_{i}, v_{i}\right) \leq f_{i}\left(u_{i}\right)-f_{i}\left(v_{i}\right)$,

(b) $p_{i}\left(v_{i}, x_{i}\right) \geq f_{i}\left(v_{i}\right)-f_{i}\left(x_{i}\right)$ for all $x_{i} \in T_{i}(v) \cup\left\{z_{i} \in X_{i}: p_{i}\left(u_{i}, z_{i}\right)>f_{i}\left(u_{i}\right)-f_{i}\left(z_{i}\right)\right\}$.

By using Theorem 3.1 again, we have the following result.

Theorem 3.10. Let $I, X_{i}, X, C_{i}, E, \theta, u, p_{i}$, and $q_{i}$ be the same as in Theorem 3.3. Suppose that there exist a nonempty compact subset $K$ of $X$ and a nonempty compact convex subset $M_{i}$ of $X_{i}$ for each $i \in I$ such that for each $y \in X \backslash K$ there exist $j \in I$ and $z_{j} \in M_{j}$ such that $q_{j}\left(u_{j}, z_{j}\right) \in-C_{j}$ and $p_{j}\left(y_{j}, z_{j}\right) \in-C_{j} \backslash\{\theta\}$. Then there exists $v=\left(v_{i}\right)_{i \in I} \in X$ such that for each $i \in I$, one has

(a) $q_{i}\left(u_{i}, v_{i}\right) \notin C_{i} \backslash\{\theta\}$,

(b) $p_{i}\left(v_{i}, x_{i}\right) \notin-C_{i} \backslash\{\theta\}$ for all $x_{i} \in\left\{z_{i} \in X_{i}: q_{i}\left(u_{i}, z_{i}\right) \in-C_{i}\right\}$.

Proof. For each $i \in I$, let $H_{i}$ and multivalued maps $F_{i}, G_{i}$, and $A_{i}$ be defined as in the proof of Theorem 3.3. For each $i \in I$, define $T_{i}: X \multimap X_{i}$ by

$$
\begin{aligned}
& T_{i}(x)=L_{i}:=\left\{z_{i} \in X_{i}: q_{i}\left(u_{i}, z_{i}\right) \in-C_{i}\right\}, \quad \forall x \in X \\
& \Longleftrightarrow T_{i}^{-}\left(z_{i}\right)= \begin{cases}X & \text { if } z_{i} \in L_{i}, \\
\emptyset & \text { if } z_{i} \in X_{i} \backslash L_{i} .\end{cases}
\end{aligned}
$$

Clearly, for each $i \in I, T_{i}^{-}\left(z_{i}\right)$ is open in $X$ for all $z_{i} \in X_{i}$. By the $C_{i}$-convexity of $q_{i}\left(u_{i}, \cdot\right), L_{i}$ is a nonempty convex subset of $X_{i}$ for all $i \in I$. Since $L_{i}$ is convex in $X_{i}$ and $L_{i} \subseteq H_{i}$, we have $\operatorname{co} T_{i}(x) \subseteq H_{i}$ for all $i \in I$. By our hypothesis, there exist a nonempty compact subset $K$ of $X$ and a nonempty compact convex subset $M_{i}$ of $X_{i}$ for each $i \in I$ such that for each $y \in X \backslash K$ there exist $j \in I$ and $z_{j} \in M_{j} \cap T_{j}(y) \cap A_{j}(y)$. Thus all the conditions of Theorem 3.1 are satisfied, and the conclusion follows from Theorem 3.1.

Remark 3.11. In Theorem 3.10, the multivalued map $T_{i}$ and the condition "for each $y \in Y$, $\operatorname{co} T_{i}(y) \subseteq\left\{x_{i} \in X_{i}: q_{i}\left(u_{i}, x_{i}\right) \notin C_{i} \backslash\{\theta\}\right\}^{\prime \prime}$ are not assumed. So Theorems 3.10 and 3.3 are different.

Theorem 3.12. Let $I, X, X, C, E, \theta$, and $p_{i}$ be the same as in Theorem 3.3. Let $u=\left(u_{i}\right)_{i \in I} \in X$ with $p_{i}\left(u_{i}, u_{i}\right)=\theta$ for all $i \in I$. Suppose that there exist a nonempty compact subset $K$ of $X$ and $a$ nonempty compact convex subset $M_{i}$ of $X_{i}$ for each $i \in I$ such that for each $y \in X \backslash K$ there exist $j \in I$ and $z_{j} \in M_{j}$ such that $p_{j}\left(u_{j}, z_{j}\right) \in-C_{j}$ and $p_{j}\left(y_{j}, z_{j}\right) \in-C_{j} \backslash\{\theta\}$. Then there exists $v=\left(v_{i}\right)_{i \in I} \in X$ such that for each $i \in I$, one has

(a) $p_{i}\left(u_{i}, v_{i}\right) \notin C_{i} \backslash\{\theta\}$,

(b) $p_{i}\left(v_{i}, x_{i}\right) \notin-C_{i} \backslash\{\theta\}$ for all $x_{i} \in\left\{z_{i} \in X_{i}: p_{i}\left(u_{i}, z_{i}\right) \in-C_{i}\right\} \cup\left\{z_{i} \in X_{i}: p_{i}\left(u_{i}, z_{i}\right) \in\right.$ $\left.C_{i} \backslash\{\theta\}\right\}$.

Proof. For each $i \in I$, let $q_{i}=p_{i}$. Applying Theorem 3.10 and following the same argument as in the proof of Theorem 3.4, one can prove the theorem.

Theorem 3.13. Let $I, X_{i}, X, C_{i}, E, \theta$, and $p_{i}$ be the same as in Theorem 3.3. Let $u=\left(u_{i}\right)_{i \in I} \in X$ with $p_{i}\left(u_{i}, u_{i}\right)=\theta$ for all $i \in I$. For each $i \in I$, let $f_{i}: X_{i} \rightarrow E$ be a $C_{i}$-v.l.s.c. and $C_{i}$-convex function. 
Suppose that there exist a nonempty compact subset $K$ of $X$ and a nonempty compact convex subset $M_{i}$ of $X_{i}$ for each $i \in I$ such that for each $y \in X \backslash K$ there exist $j \in I$ and $z_{j} \in M_{j}$ such that $f_{j}\left(z_{j}\right)-f_{j}\left(u_{j}\right) \in-C_{j}$ and $p_{j}\left(y_{j}, z_{j}\right)+f_{j}\left(z_{j}\right)-f_{j}\left(y_{j}\right) \in-C_{j} \backslash\{\theta\}$. Then there exists $v=\left(v_{i}\right)_{i \in I} \in X$ such that for each $i \in I$, one has

(a) $f_{i}\left(v_{i}\right)-f_{i}\left(u_{i}\right) \notin C_{i} \backslash\{\theta\}$,

(b) $p_{i}\left(v_{i}, x_{i}\right)+f_{i}\left(x_{i}\right)-f_{i}\left(v_{i}\right) \notin-C_{i} \backslash\{\theta\}$ for all $x_{i} \in\left\{z_{i} \in X_{i}: f_{i}\left(z_{i}\right)-f_{i}\left(u_{i}\right) \in-C_{i}\right\}$.

Corollary 3.14. Let $I, X_{i}$, and $X$ be the same as in Theorem 3.3. For each $i \in I$, let $f_{i}: X_{i} \rightarrow$ $(-\infty, \infty]$ be a l.s.c. and convex function and $p_{i}: X_{i} \times X_{i} \rightarrow(-\infty, \infty]$ be a quasi-distance. Let $u=\left(u_{i}\right)_{i \in I} \in X$ with $p_{i}\left(u_{i}, u_{i}\right)=0$ for all $i \in I$. Suppose that there exist a nonempty compact subset $K$ of $X$ and a nonempty compact convex subset $M_{i}$ of $X_{i}$ for each $i \in I$ such that for each $y \in X \backslash K$ there exist $j \in I$ and $z_{j} \in M_{j}$ such that $f_{j}\left(z_{j}\right) \leq f_{j}\left(u_{j}\right)$ and $p_{j}\left(y_{j}, z_{j}\right)<f_{j}\left(y_{j}\right)-f_{j}\left(z_{j}\right)$. Then there exists $v=\left(v_{i}\right)_{i \in I} \in X$ such that for each $i \in I$, one has

(a) $f_{i}\left(v_{i}\right) \leq f_{i}\left(u_{i}\right)$,

(b) $p_{i}\left(v_{i}, x_{i}\right) \geq f_{i}\left(v_{i}\right)-f_{i}\left(x_{i}\right)$ for all $x_{i} \in\left\{z_{i} \in X_{i}: f_{i}\left(z_{i}\right) \leq f_{i}\left(u_{i}\right)\right\}$.

Remark 3.15. [19, Theorem 4.3] is a special case of Corollary 3.14.

Theorem 3.16. Let $I, X_{i}, X, C_{i}, E, \theta$, and $p_{i}$ be the same as in Theorem 3.3. Let $u=\left(u_{i}\right)_{i \in I} \in X$ with $p_{i}\left(u_{i}, u_{i}\right)=\theta$ for all $i \in I$. For each $i \in I$, let $f_{i}: X_{i} \rightarrow E$ be a $C_{i}$-v.l.s.c. and $C_{i}$-convex function, and suppose that there exist a nonempty compact subset $K$ of $X$ and a nonempty compact convex subset $M_{i}$ of $X_{i}$ for each $i \in I$ such that for each $y \in X \backslash K$ there exist $j \in I$ and $z_{j} \in M_{j}$ such that $p_{j}\left(u_{j}, z_{j}\right)+f_{j}\left(z_{j}\right)-f_{j}\left(u_{j}\right) \in-C_{j}$ and $p_{j}\left(y_{j}, z_{j}\right)+f_{j}\left(z_{j}\right)-f_{j}\left(y_{j}\right) \in-C_{j} \backslash\{\theta\}$. Then for each $u=\left(u_{i}\right)_{i \in I} \in X$ with $p_{i}\left(u_{i}, u_{i}\right)=\theta$ for all $i \in I$, there exists $v=\left(v_{i}\right)_{i \in I} \in X$ such that for each $i \in I$, one has

(a) $p_{i}\left(u_{i}, v_{i}\right)+f_{i}\left(v_{i}\right)-f_{i}\left(u_{i}\right) \notin C_{i} \backslash\{\theta\}$,

(b) $p_{i}\left(v_{i}, x_{i}\right)+f_{i}\left(x_{i}\right)-f_{i}\left(v_{i}\right) \notin-C_{i} \backslash\{\theta\}$ for all $x_{i} \in\left\{z_{i} \in X_{i}: p_{i}\left(u_{i}, z_{i}\right)+f_{i}\left(z_{i}\right)-f_{i}\left(u_{i}\right) \in\right.$ $\left.-C_{i}\right\} \cup\left\{z_{i} \in X_{i}: p_{i}\left(u_{i}, z_{i}\right)+f_{i}\left(z_{i}\right)-f_{i}\left(u_{i}\right) \in C_{i} \backslash\{\theta\}\right\}$.

In Theorem 3.16, if $E=(-\infty, \infty]$ and $C_{i}=[0, \infty)$ for all $i \in I$, then we have the following system of Lin and Du's variant of system of Ekeland's variational principle in t.v.s.

Corollary 3.17. Let $I, X_{i}$, and $X$ be the same as in Theorem 3.3. For each $i \in I$, let $f_{i}: X_{i} \rightarrow E$ be a l.s.c. and convex function and let $p_{i}: X_{i} \times X_{i} \rightarrow(-\infty, \infty]$ be a quasi-distance. Let $u=\left(u_{i}\right)_{i \in I} \in X$ with $p_{i}\left(u_{i}, u_{i}\right)=0$ for all $i \in I$. Suppose that there exist a nonempty compact subset $K$ of $X$ and $a$ nonempty compact convex subset $M_{i}$ of $X_{i}$ for each $i \in I$ such that for each $y \in X \backslash K$ there exist $j \in I$ and $z_{j} \in M_{j}$ such that $p_{j}\left(u_{j}, z_{j}\right) \leq f_{j}\left(u_{j}\right)-f_{j}\left(z_{j}\right)$ and $p_{j}\left(y_{j}, z_{j}\right)<f_{j}\left(y_{j}\right)-f_{j}\left(z_{j}\right)$. Then for each $u=\left(u_{i}\right)_{i \in I} \in X$ with $p_{i}\left(u_{i}, u_{i}\right)=0$ for all $i \in I$, there exists $v=\left(v_{i}\right)_{i \in I} \in X$ such that for each $i \in I$, one has

(a) $p_{i}\left(u_{i}, v_{i}\right) \leq f_{i}\left(u_{i}\right)-f_{i}\left(v_{i}\right)$,

(b) $p_{i}\left(v_{i}, x_{i}\right) \geq f_{i}\left(v_{i}\right)-f_{i}\left(x_{i}\right)$ for all $x_{i} \in X_{i}$.

Remark 3.18. Corollary 3.17 generalizes [19, Theorem 4.1]. 


\section{A Vectorial Minimization Theorem and Equivalent Formulations of VSEVP}

Using Theorem 3.1 again, we also obtain an existence theorem of system of generalized vectorial equilibrium problem of the Stampacchia-type which can be regarded as a weak form of VSEVP for $C$-vectorial $\ell$-function in a Hausdorff t.v.s.

Theorem 4.1. Let $I$ be any index set. For each $i \in I$, let $X_{i}$ be a nonempty Hausdorff t.v.s. and $C_{i} \neq\{\theta\}$ be a nonempty pointed convex cone in a t.v.s. E with zero vector $\theta$. Let $X=\prod_{i \in I} X_{i}$. For each $i \in I$, let $p_{i}, q_{i}: X_{i} \times X_{i} \rightarrow E$ be $\ell_{C_{i}}$-function. Suppose that

(i) $\mathcal{L}=\left\{x=\left(x_{i}\right)_{i \in I} \in X: p_{i}\left(x_{i}, x_{i}\right)=q_{i}\left(x_{i}, x_{i}\right)=\theta\right.$ for all $\left.i \in I\right\} \neq \emptyset$,

(ii) there exist a nonempty compact subset $K$ of $X$ and a nonempty compact convex subset $M_{i}$ of $X_{i}$ for each $i \in I$ such that for each $y \in X \backslash K$ there exist $j \in I$ and $z_{j} \in M_{j}$ such that $p_{j}\left(y_{j}, z_{j}\right)+q_{j}\left(y_{j}, z_{j}\right) \in-C_{i} \backslash\{\theta\}$.

Then there exists $v=\left(v_{i}\right)_{i \in I} \in X$ such that for each $i \in I$, one has $p_{i}\left(v_{i}, x_{i}\right)+q_{i}\left(v_{i}, x_{i}\right) \notin-C_{i} \backslash\{\theta\}$ for all $x_{i} \in X_{i}$.

Proof. Let $u=\left(u_{i}\right)_{i \in I} \in \mathcal{L}$ be given. For each $i \in I$, we define $F_{i}: X \times X_{i} \multimap E, G_{i}: X \times X \times X_{i} \multimap E$ and $A_{i}: X \multimap X_{i}$ by

$$
\begin{gathered}
F_{i}\left(x, y_{i}\right)=C_{i} \backslash\{\theta\}, \quad \forall\left(x, y_{i}\right) \in X \times X_{i}, \\
G\left(t, x, y_{i}\right)=p_{i}\left(x_{i}, y_{i}\right)+q_{i}\left(x_{i}, y_{i}\right)+C_{i} \backslash\{\theta\}, \quad \forall\left(t, x, y_{i}\right) \in X \times X \times X_{i}, \\
A_{i}(x)=\left\{y_{i} \in X_{i}: \theta \in G_{i}\left(u, x, y_{i}\right)\right\}, \quad \forall x=\left(x_{i}\right)_{i \in I} \in X,
\end{gathered}
$$

respectively. Clearly,

$$
H_{i}:=\left\{x_{i} \in X_{i}: \theta \notin F_{i}\left(u, x_{i}\right)\right\}=X, \quad \forall i \in I .
$$

Using the same argument in the proof of Theorem 3.3, one can verify that all the conditions of Theorem 3.1 are satisfied. By Theorem 3.1, there exists $v=\left(v_{i}\right)_{i \in I} \in X$ such that for each $i \in I$, we have $p_{i}\left(v_{i}, x_{i}\right)+q_{i}\left(v_{i}, x_{i}\right) \notin-C_{i} \backslash\{\theta\}$ for all $x_{i} \in X_{i}$.

An existence theorem of system of generalized vector equilibrium problem is immediate from Theorem 4.1 if $q_{i} \equiv 0$ (the zero map) for all $i \in I$.

Theorem 4.2. Let $I$ be any index set. For each $i \in I$, let $X_{i}$ be a nonempty Hausdorff t.v.s., and let $C_{i} \neq\{\theta\}$ be a nonempty pointed convex cone in a t.v.s. E with zero vector $\theta$. Let $X=\prod_{i \in I} X_{i}$. For each $i \in I$, let $p_{i}: X_{i} \times X_{i} \rightarrow E$ be a $\ell_{C_{i}}$-function. Suppose that

(i) $\mathscr{H}=\left\{x=\left(x_{i}\right)_{i \in I} \in X: p_{i}\left(x_{i}, x_{i}\right)=\theta\right.$ for all $\left.i \in I\right\} \neq \emptyset$;

(ii) there exist a nonempty compact subset $K$ of $X$ and a nonempty compact convex subset $M_{i}$ of $X_{i}$ for each $i \in I$ such that for each $y \in X \backslash K$ there exist $j \in I$ and $z_{j} \in M_{j}$ such that $p_{j}\left(y_{j}, z_{j}\right) \in-C_{i} \backslash\{\theta\}$.

Then there exists $v=\left(v_{i}\right)_{i \in I} \in X$ such that for each $i \in I$, one has $p_{i}\left(v_{i}, x_{i}\right) \notin-C_{i} \backslash\{\theta\}$ for all $x_{i} \in X_{i}$. 
Remark 4.3. (a) Notice that Theorems 4.1 and 4.2 are indeed equivalent since the sum of two $\ell_{C_{i}}$-functions is also a $\ell_{C_{i}}$-function.

(b) In Theorem 4.1, the maps $p_{i}$ and $q_{i}$ are only assumed to be $\ell_{C_{i}}$-functions which need not be $C_{i}$-vectorial quasi-distances. So Theorem 4.1 is different from any theorem in Section 3 and is not a special case of any theorem in Section 3.

Theorem 4.4. Let $I, X, X, C_{i}, E$, and $\theta$ be the same as in Theorem 4.1. For each $i \in I$, let $p_{i}$ : $X_{i} \times X_{i} \rightarrow E$ be a $\ell_{C_{i}}$-function and let $f_{i}: X_{i} \rightarrow E$ be a $C_{i}$-v.l.s.c. and $C_{i}$-convex function. Suppose that

(i) $\mathscr{H}=\left\{x=\left(x_{i}\right)_{i \in I} \in X: p_{i}\left(x_{i}, x_{i}\right)=\theta\right.$ for all $\left.i \in I\right\} \neq \emptyset$,

(ii) there exist a nonempty compact subset $K$ of $X$ and a nonempty compact convex subset $M_{i}$ of $X_{i}$ for each $i \in I$ such that for each $y \in X \backslash K$ there exist $j \in I$ and $z_{j} \in M_{j}$ such that $p_{j}\left(y_{j}, z_{j}\right)+f_{j}\left(z_{i}\right)-f_{j}\left(y_{i}\right) \in-C_{j} \backslash\{\theta\}$.

Then there exists $v=\left(v_{i}\right)_{i \in I} \in X$ such that for each $i \in I$, one has $p_{i}\left(v_{i}, x_{i}\right)+f_{i}\left(x_{i}\right)-f_{i}\left(v_{i}\right) \notin-C_{i} \backslash\{\theta\}$ for all $x_{i} \in X_{i}$.

Remark 4.5. [19, Theorem 4.2] is a special case of Theorem 4.4 .

Applying Theorem 4.4, we obtain the following vectorial minimization theorem.

Theorem 4.6 (vectorial minimization theorem). Let $I, X_{i}, X, C_{i}, E$, and $\theta$ be the same as in Theorem 4.1. For each $i \in I$, let $p_{i}: X_{i} \times X_{i} \rightarrow E$ be a $\ell_{C_{i}}$-function and let $f_{i}: X_{i} \rightarrow E$ be a $C_{i}$-v.l.s.c. and $C_{i}$-convex function. Suppose that

(i) $\mathscr{d}=\left\{x=\left(x_{i}\right)_{i \in I} \in X: p_{i}\left(x_{i}, x_{i}\right)=\theta\right.$ for all $\left.i \in I\right\} \neq \emptyset$,

(ii) there exist a nonempty compact subset $K$ of $X$ and a nonempty compact convex subset $M_{i}$ of $X_{i}$ for each $i \in I$ such that for each $y \in X \backslash K$ there exist $j \in I$ and $z_{j} \in M_{j}$ such that $p_{j}\left(y_{j}, z_{j}\right)+f_{j}\left(z_{i}\right)-f_{j}\left(y_{i}\right) \in-C_{j} \backslash\{\theta\}$,

(iii) for $i \in I$ and $x_{i} \in X_{i}$ with $f_{i}\left(x_{i}\right) \notin \operatorname{Min}_{C_{i}} f_{i}\left(X_{i}\right)$, there exists $y_{i} \in X_{i}$ with $y_{i} \neq x_{i}$ such that $p_{i}\left(x_{i}, y_{i}\right)+f_{i}\left(y_{i}\right)-f_{i}\left(x_{i}\right) \in-C_{i} \backslash\{\theta\}$.

Then there exists $\widehat{x}=\left(\widehat{x}_{i}\right)_{i \in I} \in X$ such that $f_{i}\left(\widehat{x}_{i}\right) \in \operatorname{Min}_{C_{i}} f_{i}\left(X_{i}\right)$ for all $i \in I$.

Proof. Applying Theorem 4.4, there exists $v=\left(v_{i}\right)_{i \in I} \in X$ such that for each $i \in I$, we have $p_{i}\left(v_{i}, x_{i}\right)+f_{i}\left(x_{i}\right)-f_{i}\left(v_{i}\right) \notin-C_{i} \backslash\{\theta\}$ for all $x_{i} \in X_{i}$. We claim that $f_{i}\left(v_{i}\right) \in \operatorname{Min}_{C_{i}} f_{i}\left(X_{i}\right)$ for all $i \in I$. Suppose to the contrary that there exists $i_{0} \in I$ such that $f_{i_{0}}\left(v_{i_{0}}\right) \notin \operatorname{Min}_{C_{i_{0}}} f_{i_{0}}\left(X_{i_{0}}\right)$. Then, by our assumption, there exists $y_{i_{0}}=y_{i_{0}}\left(v_{i_{0}}\right) \in X_{i_{0}}$ with $y_{i_{0}} \neq v_{i_{0}}$ such that $p_{i_{0}}\left(v_{i_{0}}, y_{i_{0}}\right)+$ $f_{i_{0}}\left(y_{i_{0}}\right)-f_{i_{0}}\left(v_{i_{0}}\right) \in-C_{i_{0}} \backslash\{\theta\}$, which leads to a contradiction. Therefore $f_{i}\left(v_{i}\right) \in \operatorname{Min}_{C_{i}} f_{i}\left(X_{i}\right)$ for all $i \in I$.

The following scalar minimization theorem follows from Theorem 4.6 immediately.

Corollary 4.7. Let $I, X_{i}$, and $X$ be the same as in Theorem 4.1. For each $i \in I$, let $f_{i}: X_{i} \rightarrow(-\infty, \infty]$ be a l.s.c. and convex function and let $p_{i}: X_{i} \times X_{i} \rightarrow(-\infty, \infty]$ be a $\ell$-function. Suppose that

(i) $\Phi=\left\{x=\left(x_{i}\right)_{i \in I} \in X: p_{i}\left(x_{i}, x_{i}\right)=0\right.$ for all $\left.i \in I\right\} \neq \emptyset$,

(ii) there exist a nonempty compact subset $K$ of $X$ and a nonempty compact convex subset $M_{i}$ of $X_{i}$ for each $i \in I$ such that for each $y \in X \backslash K$ there exist $j \in I$ and $z_{j} \in M_{j}$ such that $p_{j}\left(y_{j}, z_{j}\right)<f_{j}\left(y_{i}\right)-f_{j}\left(z_{i}\right)$, 
(iii) for any $i \in I$ and $x_{i} \in X_{i}$ with $f_{i}\left(x_{i}\right)>\inf _{z_{i} \in X_{i}} f_{i}\left(z_{i}\right)$ there exists $y_{i} \in X_{i}$ with $y_{i} \neq x_{i}$ such that $p_{i}\left(x_{i}, y_{i}\right)<f_{i}\left(x_{i}\right)-f_{i}\left(y_{i}\right)$.

Then there exists $\widehat{x}=\left(\widehat{x}_{i}\right)_{i \in I} \in X$ such that $f_{i}\left(\widehat{x}_{i}\right)=\inf _{z_{i} \in X_{i}} f_{i}\left(z_{i}\right)$ for all $i \in I$.

Remark 4.8. (a) [19, Theorem 5.5] is a special case of Corollary 4.7.

(b) Theorems 4.4 and 4.6 are equivalent if they further add the condition "for each $i \in I, p_{i}\left(x_{i}, y_{i}\right) \in C_{i}$ for all $x_{i}, y_{i} \in X_{i}$." Indeed, it suffices to show that Theorem 4.6 implies Theorem 4.4. Suppose that for each $x=\left(x_{i}\right)_{i \in I} \in X$, there exists $i_{x} \in I$ such that $p_{i_{x}}\left(x_{i_{x}}, y_{i_{x}}\right)+$ $f_{i_{x}}\left(y_{i_{x}}\right)-f_{i_{x}}\left(x_{i_{x}}\right) \in-C_{i_{x}} \backslash\{\theta\}$ for some $y_{i_{x}} \in X_{i_{x}}$ with $y_{i_{x}} \neq x_{i_{x}}$. Then, by Theorem 4.6, there exists $v=\left(v_{i}\right)_{i \in I} \in X$ such that $f_{i}\left(v_{i}\right) \in \operatorname{Min}_{C_{i}} f_{i}\left(X_{i}\right)$ or

$$
f_{i}\left(x_{i}\right)-f_{i}\left(v_{i}\right) \notin-C_{i} \backslash\{\theta\}, \quad \forall x_{i} \in X_{i}, \forall i \in I .
$$

But from our assumption, there exists $i_{v} \in I$ such that

$$
p_{i_{v}}\left(v_{i_{v}}, w_{i_{v}}\right)+f_{i_{v}}\left(w_{i_{v}}\right)-f_{i_{v}}\left(v_{i_{v}}\right) \in-C_{i} \backslash\{\theta\}
$$

for some $w_{i_{v}} \in X_{i_{v}}$ with $w_{i_{v}} \neq v_{i_{v}}$. It follows that

$$
f_{i_{v}}\left(w_{i_{v}}\right)-f_{i_{v}}\left(v_{i_{v}}\right) \in-p_{i_{v}}\left(v_{i_{v}}, w_{i_{v}}\right)-C_{i} \backslash\{\theta\} \subseteq-C_{i} \backslash\{\theta\},
$$

which leads to a contradiction. follows.

Now, we give some equivalent formulations of Theorem 4.1 (when $I$ is a singleton) as

Theorem 4.9. Let $X$ be a Hausdorff t.v.s., let $C \neq\{\theta\}$ be a nonempty pointed convex cone in a t.v.s. $E$ with zero vector $\theta, p, q: X \times X \rightarrow E$ be $\ell_{C}$-functions. Suppose that

(1) $\mathcal{R}=\{x \in X: p(x, x)=q(x, x)=0\} \neq \emptyset$,

(2) there exist a nonempty compact subset $K$ of $X$ and a nonempty compact convex subset $M$ of $X$ such that for each $y \in X \backslash K$ there exists $z \in M$ such that $p(y, z)+q(y, z) \in-C \backslash\{\theta\}$.

Then the following statements are equivalent.

(i) (Vectorial Variant of Ekeland's Variational Principle). There exists $v \in X$ such that $p(v, x)+q(v, x) \notin-C \backslash\{\theta\}$ for all $x \in X$.

(ii) (Common Fixed Point Theorem for a Family of Multivalued Maps). Let $\Lambda$ be an index set. For each $i \in \Lambda$, let $T_{i}: X \multimap X$ be a multivalued map with nonempty values such that for each $(i, x) \in \Lambda \times X$ with $x \notin T_{i}(x)$, there exists $y=y(x, i) \in X$ with $y \neq x$ such that

$$
p(x, y)+q(x, y) \in-C \backslash\{\theta\}
$$

Then there exists $x_{0} \in X$ such that $x_{0} \in \bigcap_{i \in \Lambda} T_{i}\left(x_{0}\right)$. That is, $\left\{T_{i}\right\}_{i \in \Lambda}$ has a common fixed point in $X$. 
(iii) (Common Fixed Point Theorem for a Family of Single-Valued Maps). Let $\Lambda$ be an index set. For each $i \in \Lambda$, suppose that $T_{i}: X \rightarrow X$ is a self-map satisfying

$$
p\left(x, T_{i} x\right)+q\left(x, T_{i} x\right) \in-C \backslash\{\theta\} .
$$

for all $x \neq T_{i}(x)$. Then there exists $x_{0} \in X$ such that $T_{i} x_{0}=x_{0}$ for all $i \in \Lambda$;

(iv) (Maximal Element Theorem for a Family of Multivalued Maps). Let $\Lambda$ be an index set. For each $i \in \Lambda$, let $T_{i}: X \multimap X$ be a multivalued map. Suppose that for each $(i, x) \in \Lambda \times X$ with $T_{i}(x) \neq \emptyset$, there exists $y=y(x, i) \in X$ with $y \neq x$ such that

$$
p(x, y)+q(x, y) \in-C \backslash\{\theta\}
$$

Then there exists $x_{0} \in X$ such that $T_{i}\left(x_{0}\right)=\emptyset$ for all $i \in \Lambda$.

Proof. By Theorem 4.1, conclusion (i) holds.

(a) "(i) $\Longleftrightarrow(i i) "$

$(\Rightarrow)$ By (i), there exists $v \in X$ such that $p(v, x)+q(v, x) \notin-C \backslash\{\theta\}$ for all $x \in X$. We claim that $v \in T_{i}(v)$ for all $i \in I$. If $v \notin T_{i_{0}} v$ for some $i_{0} \in I$, then, by hypothesis, there exists $w\left(v, i_{0}\right) \in X$ with $w\left(v, i_{0}\right) \neq v$ such that

$$
p\left(v, w\left(v, i_{0}\right)\right)+q\left(v, w\left(v, i_{0}\right)\right) \in-C \backslash\{\theta\},
$$

which leads to a contradiction. Hence $v \in \bigcap_{i \in I} T_{i}(v)$ and $v$ is a common fixed point of $\left\{T_{i}\right\}_{i \in I}$.

$(\Leftarrow)$ Suppose that for each $x \in X$, there exists $y \in X$ with $y \neq x$ such that $p(x, y)+$ $q(x, y) \in-C \backslash\{\theta\}$. Then for each $x \in X$, we can define a multivalued map $T: X \multimap X \backslash\{\emptyset\}$ by

$$
T(x)=\{y \in X: p(x, y)+q(x, y) \in-C \backslash\{\theta\}\} .
$$

Clearly, $x \notin T(x)$ for all $x \in X$. But, by (ii), $T$ has a fixed point $v$ in $X$, a contradiction. So (i) is true.

(b) "(ii) $\Longleftrightarrow($ iii $) "$

$(\Rightarrow)$ Suppose (ii) holds. Under the assumption of (iii), for each $i \in I$, let $\varphi_{i}: X \multimap X$ be defined by $\varphi_{i}(x)=\left\{T_{i}(x)\right\}$. Then for each $(i, x) \in I \times X$ with $x \notin \varphi_{i}(x)$, we have $x \neq T_{i}(x)$. By hypothesis of (iii), $p\left(x, T_{i} x\right)+q\left(x, T_{i} x\right) \in-C \backslash\{\theta\}$. Therefore, by (ii), there exists $v \in X$ such that $v \in \bigcap_{i \in I} \varphi_{i}(v)$ or $T_{i} v=v$ for all $i \in I$, and hence (iii) is proved.

$(\Leftarrow)$ Assume (iii) holds. Under the assumption of (ii), for each $(i, x) \in I \times X$ with $x \notin T_{i}(x)$, there exists $y(x, i) \in X$ with $y(x, i) \neq x$ such that

$$
p(x, y(x, i))+q(x, y(x, i)) \in-C \backslash\{\theta\} .
$$


Define $\tau_{i}: X \rightarrow X$ by

$$
\tau_{i}(x)= \begin{cases}x & \text { if } x \in T_{i}(x), \\ y(x, i) & \text { if } x \notin T_{i}(x) .\end{cases}
$$

Hence $\tau_{i}$ is a self-map of $X$ into $X$ satisfying $p\left(x, \tau_{i}(x)\right)+q\left(x, \tau_{i}(x)\right) \in-C \backslash\{\theta\}$ for all $x \neq \tau_{i}(x)$. By (iii), there exists $v \in X$ such that $v=\tau_{i}(v) \in T_{i}(v)$ for all $i \in I$. This shows that (iii) implies (ii).

(c) $"(i) \Longleftrightarrow(i v)^{\prime \prime}$

$\Leftrightarrow)$ Using (i), there exists $v \in X$ such that $p(v, x)+q(v, x) \notin-C \backslash\{\theta\}$ for all $x \in X$. We want to show that $T_{i}(v)=\emptyset$ for all $i \in I$. Suppose to the contrary that there exists $i_{0} \in I$ such that $T_{i_{0}}(v) \neq \emptyset$. By hypothesis of (iv), there exists $w=w\left(v, i_{0}\right) \in X$ with $w \neq v$ such that

$$
p(v, w)+q(v, w) \in-C \backslash\{\theta\},
$$

which is a contradiction. Therefore $T_{i}(v)=\emptyset$ for all $i \in I$.

$(\Leftarrow)$ Suppose that for each $x \in X$, there exists $y \in X$ with $y \neq x$ such that $p(x, y)+$ $q(x, y) \in-C \backslash\{\theta\}$. For each $x \in X$, define a multivalued map $T: X \multimap X \backslash\{\emptyset\}$ by

$$
T(x)=\{y \in X: p(x, y)+q(x, y) \in-C \backslash\{\theta\}\} .
$$

Then $T(x) \neq \emptyset$ for all $x \in X$. But applying (iv), there exists $x_{0} \in X$ such that $T\left(x_{0}\right)=\emptyset$, a contradiction. Hence (i) holds.

Remark 4.10. Theorem 4.9 improves and generalizes Theorems 4.2, 5.1, 5.2, 5.3, and 5.4 in [19].

\section{Acknowledgment}

This research was supported partially by grant no. NSC 99-2115-M-017-001 of the National Science Council of the Republic of China.

\section{References}

[1] E. Blum and W. Oettli, "From optimization and variational inequalities to equilibrium problems," The Mathematics Student, vol. 63, no. 1-4, pp. 123-145, 1994.

[2] Q. H. Ansari, S. Schaible, and J.-C. Yao, “The system of generalized vector equilibrium problems with applications," Journal of Global Optimization, vol. 22, no. 1-4, pp. 3-16, 2002.

[3] G. Isac, V. A. Bulavsky, and V. V. Kalashnikov, Complementarity, Equilibrium, Efficiency and Economics, vol. 63 of Nonconvex Optimization and Its Applications, Kluwer Academic Publishers, Dordrecht, The Netherlands, 2002.

[4] L.-J. Lin, "System of generalized vector quasi-equilibrium problems with applications to fixed point theorems for a family of nonexpansive multivalued mappings," Journal of Global Optimization, vol. 34, no. 1, pp. 15-32, 2006.

[5] I. Ekeland, "Sur les problèmes variationnels," Comptes Rendus de l'Académie des Sciences, Série A-B, vol. 275, pp. A1057-A1059, 1972. 
[6] I. Ekeland, "On the variational principle," Journal of Mathematical Analysis and Applications, vol. 47, pp. 324-353, 1974.

[7] I. Ekeland, "Nonconvex minimization problems," Bulletin of the American Mathematical Society, vol. 1, no. 3, pp. 443-474, 1979.

[8] G.-Y. Chen, X. Huang, and X. Yang, Vector Optimization, vol. 541 of Lecture Notes in Economics and Mathematical Systems, Springer, Berlin, Germany, 2005.

[9] G. Y. Chen, X. Q. Yang, and H. Yu, "A nonlinear scalarization function and generalized quasi-vector equilibrium problems," Journal of Global Optimization, vol. 32, no. 4, pp. 451-466, 2005.

[10] W.-S. Du, "On some nonlinear problems induced by an abstract maximal element principle," Journal of Mathematical Analysis and Applications, vol. 347, no. 2, pp. 391-399, 2008.

[11] W.-S. Du, "Hybrid inclusion and disclusion systems with applications to equilibria and parametric optimization," Journal of Global Optimization, vol. 47, no. 1, pp. 119-132, 2010.

[12] A. Göpfert, H. Riahi, C. Tammer, and C. Zălinescu, Variational Methods in Partially Ordered Spaces, CMS Books in Mathematics/Ouvrages de Mathématiques de la SMC, 17, Springer, New York, NY, USA, 2003.

[13] A. Göpfert, Chr. Tammer, and C. Zălinescu, “On the vectorial Ekeland's variational principle and minimal points in product spaces," Nonlinear Analysis: Theory, Methods E Applications, vol. 39, no. 7, pp. 909-922, 2000.

[14] A. H. Hamel, "Phelps' lemma, Daneš' drop theorem and Ekeland's principle in locally convex spaces," Proceedings of the American Mathematical Society, vol. 131, no. 10, pp. 3025-3038, 2003.

[15] A. Hamel and A. Löhne, "A minimal point theorem in uniform spaces," in Nonlinear Analysis and Applications: To V. Lakshmikantham on his 80th Birthday. Vol. 1, 2, pp. 577-593, Kluwer Academic Publishers, Dordrecht, The Netherlands, 2003.

[16] D. H. Hyers, G. Isac, and T. M. Rassias, Topics in Nonlinear Analysis \& Applications, World Scientific, River Edge, NJ, USA, 1997.

[17] L.-J. Lin and W.-S. Du, "Ekeland's variational principle, minimax theorems and existence of nonconvex equilibria in complete metric spaces," Journal of Mathematical Analysis and Applications, vol. 323, no. 1, pp. 360-370, 2006.

[18] L.-J. Lin and W.-S. Du, "Some equivalent formulations of the generalized Ekeland's variational principle and their applications," Nonlinear Analysis: Theory, Methods E Applications, vol. 67, no. 1, pp. 187-199, 2007.

[19] L.-J. Lin and W.-S. Du, "Systems of equilibrium problems with applications to new variants of Ekeland's variational principle, fixed point theorems and parametric optimization problems," Journal of Global Optimization, vol. 40, no. 4, pp. 663-677, 2008.

[20] L.-J. Lin and W.-S. Du, "On maximal element theorems, variants of Ekeland's variational principle and their applications," Nonlinear Analysis: Theory, Methods E Applications, vol. 68, no. 5, pp. $1246-$ 1262, 2008.

[21] L.-J. Lin and C.-S. Chuang, "Existence theorems for variational inclusion problems and the set-valued vector Ekeland variational principle in a complete metric space," Nonlinear Analysis: Theory, Methods $\mathcal{E}$ Applications, vol. 70, no. 7, pp. 2665-2672, 2009.

[22] W. Oettli and M. Théra, "Equivalents of Ekeland's principle," Bulletin of the Australian Mathematical Society, vol. 48, no. 3, pp. 385-392, 1993.

[23] Chr. Tammer, "A generalization of Ekeland's variational principle," Optimization, vol. 25, no. 2-3, pp. $129-141,1992$.

[24] C. D. Aliprantis and K. C. Border, Infinite-Dimensional Analysis, Springer, Berlin, Germany, 2nd edition, 1999.

[25] N. X. Tan, "Quasivariational inequalities in topological linear locally convex Hausdorff spaces," Mathematische Nachrichten, vol. 122, pp. 231-245, 1985. 


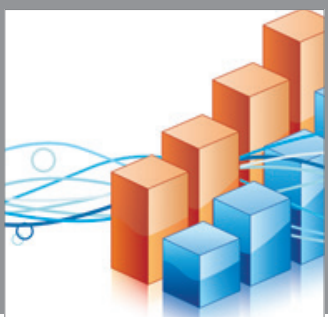

Advances in

Operations Research

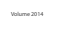

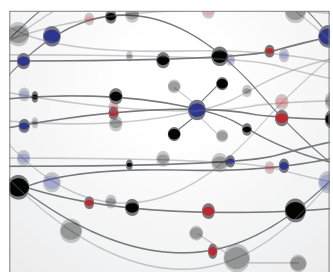

\section{The Scientific} World Journal
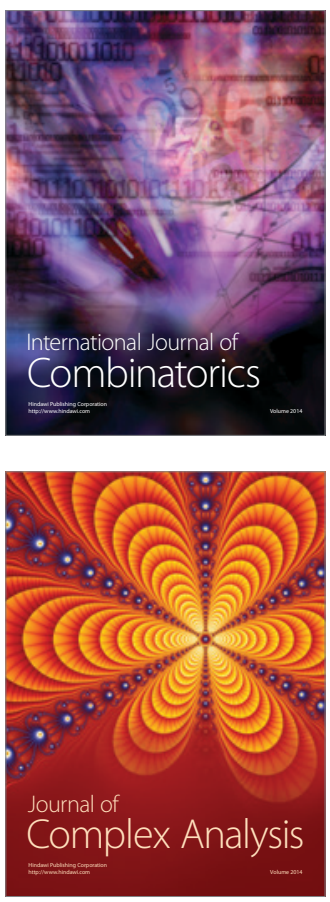

International Journal of

Mathematics and

Mathematical

Sciences
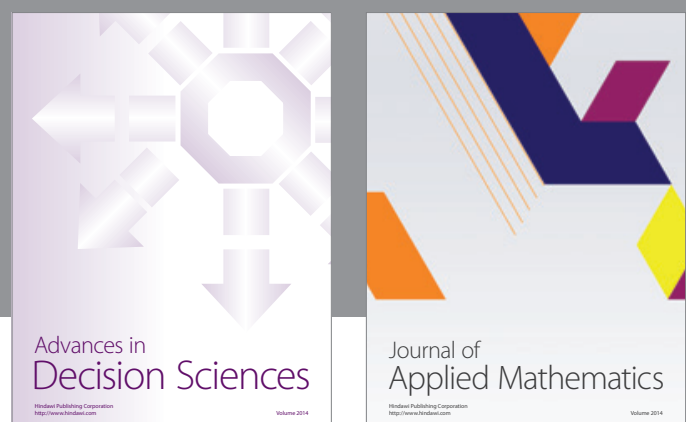

Journal of

Applied Mathematics
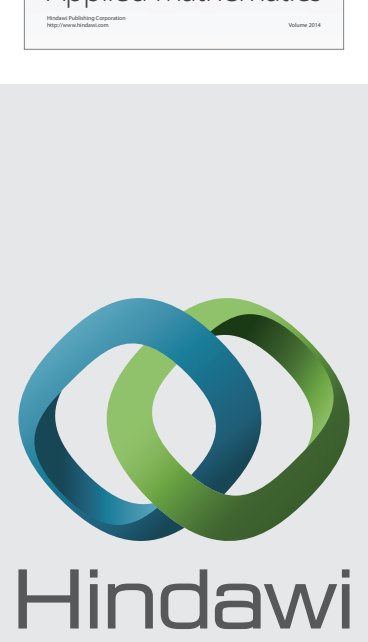

Submit your manuscripts at http://www.hindawi.com
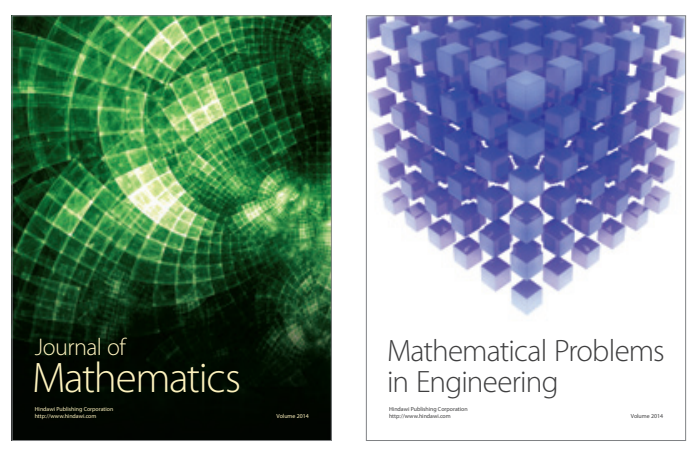

Mathematical Problems in Engineering
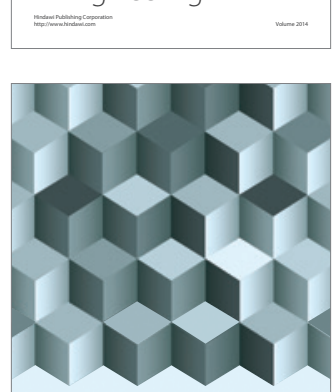

Journal of

Function Spaces
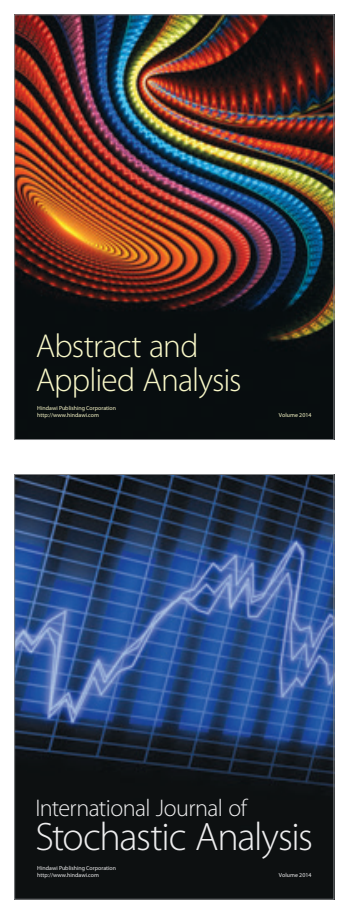

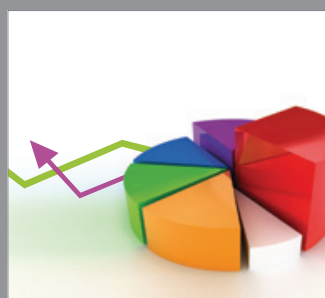

ournal of

Probability and Statistics

Promensencen
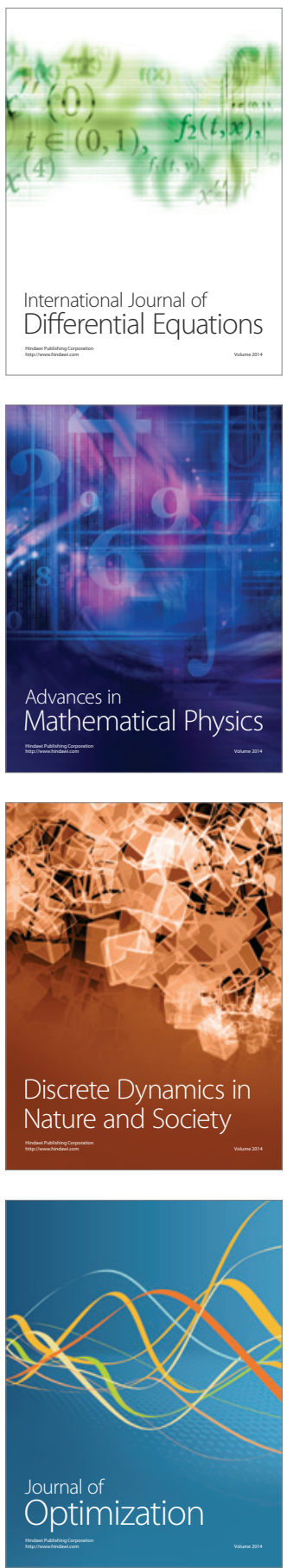